\title{
Las infotecnologías, su aplicación en la formación de la cultura ambiental
}

\author{
As infotecnologias, sua aplicação na formação da cultura ambiental \\ Infotechnologies, their application in the formation of environmental culture
}

\author{
Alcides Antúnez Sánchez* \\ Eduardo Diaz Ocampo*
}

\section{Resumen}

\begin{abstract}
El artículo analiza las debilidades, y oportunidades que aportan las infotecnologias en Cuba aplicadas en la formación ambiental en las formas de gestión en el proceso de actualización el modelo económico social. Se realiza un análisis crítico de su incidencia jurídica, que permita a futuro a la Administración Pública alcanzar las metas ambientales en aras de potenciar en la nación cubana la protección del bien público ambiente por la multidisciplinariedad de la temática. Ello permitirá alcanzar prosperidad y el equilibrio con el bien público ambiente para lograr alcanzar el desarrollo sostenible a través de la empresa responsable con el ambiente. Se han utilizado métodos científicos en el desarrollo de la investigación como el histórico, el de análisis y síntesis, el de inducción deducción.
\end{abstract}

Palabras Ilaves: Sujetos de gestión. Empresa responsable. Competencia. Metas ambientales.

\section{Resumo}

O artigo analisa as fraquezas e oportunidades oferecidas pelas infotecnologias aplicadas em treinamento ambiental em Cuba nas formas de gestão no processo de atualização do modelo econômico social. Realiza-se uma análise crítica do seu impacto legalque permite que a Administração Pública no futuro atinja metas ambientais para promover a proteção do bem público na nação cubana através da natureza multidisciplinar do assunto. Isto nos permitirá alcançar prosperidade e equilíbrio no ambiente público para o desenvolvimento sustentável por meio da empresa responsável pelo meio ambiente. Métodos científicos foram utilizados no desenvolvimento da pesquisa, tais como: histórico; análise e síntese; e dedução e indução.

Palavras-chave: Assuntos de gestão. Empresa responsável. Concorrência. Metas ambientais.

\section{Abstract}

The article analyzes the weaknesses and opportunities offered by info-technologies applied in Cuba in environmental training in forms of management in the process of updating the social economic model. A critical analysis of its legal impact is carried out allowing the Public Administration to achieve environmental goals in order to promote the protection of the good public environment in the Cuban nation by the multidisciplinary nature of the issue. This will allow us to achieve prosperity and balance with the good public environment to achieve sustainable development through the responsible company with the environment. Scientific methods have been used in the development of research as the historical, the analysis and synthesis, the induction deduction.

Keywords: Management subjects. Responsible company. Competition. Environmental goals.

Máster en Derecho de la Empresa. Profesor Auxiliar Derecho Ambiental e Internacional. Departamento de Derecho. Facultad de Ciencias Económicas y Sociales, Universidad de Granma. Granma - Cuba. E-mail: aantunez@udg.co.cu.

Doctor en Jurisprudencia. Licenciado en Ciencias de la Educación. Licenciado en Ciencias Económicas, Políticas y Sociales. Abogado de los Tribunales de la Republica del Ecuador. Magister en Desarrollo Curricular. Professor de Derecho Laboral y Derecho Constitucional. Rector da Universidad Técnica Estatal de Quevedo. Quevedo - Ecuador. E-mail: ediaz@uteq.edu.ec. 


\section{Introduccion}

La preparación de los ciudadanos de un país es una de las necesidades más importantes a satisfacer por la Administración Pública y, ello lo convierte en uno de sus problemas esenciales, máxime si es reconocido como un principio constitucional, como es el caso de Cuba. Es un elemento objetivo, que na nación requiere que sus miembros posean el nivel cultural que le posibilite desarrollar sus labores de forma eficiente y eficaz.

Para ello, las universidades actuales, y en especial las universidades cubanas, poseen la responsabilidad de incidir no sólo en el traslado de los avances científicos al sector productivo en las formas de gestión, sino incidir a la vez, en los mejores métodos y vías para el logro de la formación continua y necesaria en los graduados en la materia ambiental, y es aquí donde con la aplicación de las infotecnologías incidirán en dotar de estas habilidades a los graduados universitarios para lograr alcanzar las metas ambientales, en respuesta a los principios constitucionales.

Esta afirmación, permite ponderar que la Universidad del Siglo XXI deba ser vista como una organización socialmente activa, abierta e interconectada con su entorno y en la cual se formen individuos portadores de una cultura de aprendizaje continuo, capaces de actuar en ambientes intensivos en información, mediante un uso racional de las nuevas tecnologías de la información y las comunicaciones.

Todo lo anterior conlleva a que la formación de los trabajadores en las formas de gestión cubanas, sea continua y necesaria, la misma permitirá que los avances de la ciencia y la técnica se implementen a través de su socialización para garantizar la competitividad de las empresas como un valor añadido; es una responsabilidad de las empresas, y debe ser gestionada para alcanzar las metas ambientales como empresas responsables con el ambiente no como un gasto sino como una inversión. En la actualidad al éxito empresarial se asocia al concepto de la "empresa educadora, organización que aprende o the learning organization".

Nos dice, que el éxito de la formación empresarial está muy ligado al logro de implementar en la organización la capacidad de "aprender a aprender" y es dependiente de la filosofía organizacional existente y, en particular, de los valores que se formen en los trabajadores, en este ensayo se pondera el "valor ambiental".

La formación es uno de los elementos que conformarán a los recursos humanos en "la ventaja competitiva básica" de las empresas de este siglo XXI. El nivel de preparación y el nivel de gestión de esos recursos humanos, determinarán la referida ventaja competitiva. Por estas razones, se centrará el objetivo del ensayo en fundamentar la necesidad de empoderar a las formas de gestión cubanas en el conocimiento y aplicación de las Tics, como una necesidad para tributar al cumplimiento de las metas ambientales y con ello alcanzar el desarrollo sostenible como pieza clave. Fueron utilizados como métodos en el desarrollo del ensayo, el histórico-lógico, el de análisis-síntesis, deducción-inducción, permitió el estudio detallado de la gestión de contenido y su evolución. No está asociado a ningún proyecto de financiamiento, siendo una creación libre de los autores.

\section{Las infotecnologías, necesidad de su aplicación dentro de las formas de gestión}

Con el surgimiento de Internet en 1991, en el siglo pasado como un hecho social, indudablemente marcó un hito histórico en el desarrollo de la sociedad y la tecnología de la información; ya en el año 1996, se precia como se definió como una nueva profesión enmarcada en la Ciencias de la información, la de infotecnológo, la cual surge a partir de la evolución de las nuevas tecnologías de la información y las comunicaciones (TICs). Como parte del proceso de evolución sociedad- tecnología.

Por consiguiente, la Infotecnología deja de ser una de las disciplinas de la Bibliotecología y la Documentación para convertirse en un componente esencial de la cultura básica de los profesores y estudiantes universitarios. Esta nueva realidad impone la exigencia de desarrollar nuevos métodos de 
búsqueda, introducir los sistemas que tratan de agregar información semántica a los documentos, nuevas formas de indización y nuevos servicios. Por otra parte, la necesidad de facilitar el uso de las herramientas computacionales desencadena una amplia actividad investigadora en torno a interfaces del usuario.

En este ir y venir, Sebastia (1996) identificaba a las tecnologías de la información como: "[...] un nuevo perfil y una nueva nomenclatura [...]" y a su vez propuso, para designar a la formación de los profesionales y de los usuarios de la información, el término: infotecnologías, pero no es hasta el año 2001, cuando se consolida todo este desarrollo tecnológico, integrándose con todas las esferas de la sociedad.

En este mismo plano, se constata como en el año 1999, se fija el inicio de la nueva Internet, conocida como la Web 2.0 por DiNucci (1999), la que tiene como premisa tecnológica el soporte de la Red Universal Digital (RUD), según esta autora, los primeros atisbos de la Web 2.0 pueden verse en lo que es tan sólo el primer peldaño de un futuro más o menos inmediato, muy superior a la Web 1.0 por su relevancia y oportunidad para los negocios al ser sus protagonistas sus usuarios. La web será entendida no simplemente como pantallas de texto e imagen, sino más bien como un sistema de transporte de información y conocimiento, el éter del que derivará la interactividadaunque algunos autores prefieran la denominación de Nuevo Entorno Tecno social (NET) el cual conforma la nueva versión de Internet y adquiere popularidad en el 2005: "[...] Este Nuevo Entorno supondría una singular novedad en la breve pero acelerada historia de la Era de la Información, a la que se ha llegado, al menos parcialmente, gracias a un proceso de evolución sociedad-tecnología o infotecnologías [...]." (CABERO ALMANERA, 2007; SANTIAGO CAMPEÓN; NAVARIDAS NALDA, 2012; SOLER PELLICER, 2015).

Dentro del desarrollo evolutivo de las Tics, se aprecia como las Infotecnologías, como parte de la "cultura tecnológica", entendida por la cultura como un conjunto de "conocimientos y habilidades prácticas" de los individuos, son herramientas indispensables para mantener relaciones exitosas con el nuevo entorno y con otros individuos, al ser una página digital dinámica. (GOSSMAN, 2006; FUMERO, ROCA; SÁEZVACAS, 2007; CABERO ALMANERA, 2009).

¿Por qué? es un hecho social que el ambiente telemático se configura a partir de la irrupción de la sociedad en las Tics. Donde la barrera de espacio y tiempo propia del ser humano se rompe, originando una nueva forma de enfrentarse y entender la propia existencia y la interacción con los otros y con lo otro. La llamada "sociedad de la información y el conocimiento", ante la generación de un volumen insospechado de información en el mundo empresarial, constituye hoy un reto para los profesionales, la que deberá utilizarse con responsabilidad, siendo esta parte una preocupación para el control público ejecutado por la Administración Pública en los Estados.

Se precia que la tecnología digital, permite la creación de entornos flexibles para el aprendizaje y favorecen el trabajo colaborativo entre los educandos, rompiendo con los escenarios formativos tradicionales, con ello se ofrecen nuevas posibilidades para las orientaciones y la tutoría de los alumnos y facilitar una formación permanente. Todos estos espacios telemáticos, permiten crear entornos de comunicación sincrónicos y asincrónicos, facilitan eliminar las barreras espacio-temporales entre las personas, potencian contextos interactivos entre los usuarios (SALINAS, 2002; CARABANTES, 2005; CABERO ALMANERA, 2008).

Empero, ¿Para qué son necesarias las herramientas de la informática? A partir de las visiones conclusivas resultantes de la Cumbre Mundial de la "sociedad de la información" (2003), y la Declaración de Principios de Ginebra, adoptada por los gobiernos, en la cual se expresa:

\footnotetext{
Nosotros... declaramos nuestro deseo y compromiso comunes de construir una 'sociedad de la información' centrada en la persona, integradora y orientada al desarrollo, en la que todos puedan crear, consultar, utilizar y compartir la información y conocimiento para que las personas, las comunidades y los pueblos puedan emplear plenamente sus posibilidades en la promoción de su desarrollo sostenible y en la mejora de su calidad de vida, sobre la base de los propósitos y principios de la Carta de las Naciones Unidas; la que muestra el respeto pleno y defensa de la Declaración Universal de Derechos Humanos (1949), donde se reconocen los derechos de $4^{\text {ta }}$ generación. (SANZ LARRUGA, 1996, p. 111).
} 
En esta línea de análisis, entrar en este "mundo socio tecnológico", requiere la formación de la población en general con ciertas habilidades en el uso de las Tics, parten de educar colectivamente a los beneficiarios de estas tecnologías como factores del crecimiento, igualdad e inclusión social, donde entra a jugar un rol esencial los ciudadanos; para su irradiación podrá aplicarse el modelo de la triple hélice, pero se estima que son necesarios los conocimientos básicos para dominar estas tecnologías, y las habilidades adquiridas para poder asimilarlas e implementarlas (SÁNCHEZ RODRÍGUEZ, 2005; ANTÚNEZ SÁNCHEZ, 2016).

No obstante, el siglo XXI le muestra nuevos retos para la enseñanza superior al mundo, uno de estos en la transformación del proceso de enseñanza aprendizaje. Autores como Duart y Sangrá (2000), han señalado que el uso de las Tics en los espacios universitarios, permite el desarrollo de tres elementos a quienes lo utilizan, con mayor flexibilidad e interactividad; por esto la vinculación con los docentes y el resto del alumnado permiten mayor colaboración y participación, y la facilidad para acceder a los materiales de estudio y a otras fuentes complementarias de información, y es aquí donde aparece el EVA.

Cabero Almanera y Llorente (2005) reseñaron que el uso de las tecnologías en los centros educativos, favorece en los educandos los medios para la adquisición de las destrezas tecnológicas, las que se requieren en la actual sociedad de la información y del conocimiento, también han notificado que se amplía el acceso al aprendizaje, el que se mejorará con la calidad de la enseñanza, donde se aprecia el desarrollo y expansión de algunas tecnologías en este sector. Para alcanzar la formación integral humanista, se toman como base los pilares de la educación presentados en la UNESCO (1996) por la comisión internacional sobre la educación para el siglo XXI, en los cuales se hacen explícitos cuatro dimensiones del aprendizaje humano: aprender a conocer, aprender a hacer, aprender a ser, aprender a vivir juntos. El exponer este propósito no implica la obtención del resultado, la formación humanista como una meta a alcanzar requiere de la concepción de la idea, su planificación; pero exige de su concreción en acciones específicas concretas, factibles, evaluables conducentes a alcanzar las metas diseñadas.

A juicio de los autores, por su experiencia en la formación de profesionales, en especial en la materia ambiental y la informática, se precia que la formación que brindan nuestras universidades es distintiva por su carácter humanista en comparación con la de los países latinoamericanos en general, empero debe fortalecerse la formación en materia ambiental que tribute con ello a alcanzar el desarrollo sostenible, tal y como se reconoce en el texto constitucional patrio, en el artículo 27.

No deja de ser un hecho veraz, que el porqué de la preparación de los ciudadanos de un país es una de las necesidades más importantes a satisfacer en cualquier sociedad, lo que se convierte en un problema esencial de la misma. Una nación moderna requiere que todos sus miembros posean cierto nivel cultural que le posibilite desarrollar sus labores de forma eficiente. Aquel país en el que todos sus ciudadanos ejecutan sus labores a un nivel de excelencia, es una nación preparada y puede ocupar un lugar de vanguardia en el concierto universal de los Estados.

Un individuo preparado, puede enfrentarse a los problemas que se le presentan en su puesto de trabajo y los resuelve de manera adecuada. De ese modo, el concepto preparación se convierte en el punto de partida de la pedagogía como ciencia, a la vez que en una categoría de la misma. Para satisfacer la necesidad de la preparación de los ciudadanos de una sociedad hace falta formarlos, es el caso de la materia ambiental. No es un secreto, que el mundo laboral en su evolución ha dejado atrás la organización tradicional caracterizada por un sin número de tareas repetitivas, trabajos en línea, toma de decisiones centralizada, etcétera. La actual situación requiere de empresas dinámicas y con altos estándares de calidad que puedan adaptarse fácilmente a un entorno cambiante y exigente, en el que va a ser fundamental la capacidad para adaptarse al mercado y dar respuesta a las nuevas demandas de atención o satisfacción al cliente de forma individualizada y personalizada. Esta nueva sociedad ha generado, como consecuencia de todo lo anterior, un nuevo concepto de perfiles profesionales y de competencias y cualificaciones que se hallan caracterizadas, entre otros aspectos, precisamente por esa capacidad de adaptación. 
Uno de los elementos más importantes y de impulso al desarrollo científico y tecnológico, consiste en garantizar la superación continua de los recursos humanos que han de llevarlo a cabo, especialmente en aquellas personas dedicadas al ejercicio de la investigación, la creación científico técnica y la innovación. Por lo tanto, la formación continua de las personas son tareas de primer orden para la sociedad contemporánea, donde una de las herramientas a explotar son las Tics, es el caso de la Empresa de Alta Tecnología y las instituciones académicas, a través de la economía basada en el conocimiento (ANTÚNEZ SÁNCHEZ, 2016).

Para Brunner (1995), la generación de conocimientos, su diseminación y utilización, han llegado a ser un factor clave para el desarrollo y competitividad de las naciones; incluso más importante, que los recursos naturales, el trabajo abundante o el capital financiero.

Todo lo anterior conlleva a que la formación de los trabajadores, en esa formación continua necesaria, la misma que permite introducir los avances de la ciencia y la técnica (DAVENPORT, 1998) y garantizar la competitividad de las empresas, es una responsabilidad de las empresas, y debe ser gestionada por esta como una inversión a largo plazo (CHIAVENATO, 2009).

En la actualidad el éxito empresarial, se pondera como está ligado el concepto de la "empresa educadora", o la "organización que aprende" o "the learning organization". La formación o educación empresarial está muy ligada a "aprender a aprender", a la filosofía organizacional y, en particular, a los valores. En todo caso, como acertadamente se advierte por Alles (2012), hay que considerar que si bien las distintas generaciones tienen unas características que definen el comportamiento de los individuos que las conforman, no es menos cierto que estos comportamientos pueden ser diferentes en función de los hábitos y comportamientos de los individuos de forma que, por ejemplo, una persona que por edad formaría parte de la denominada "Generación X", la inmediatamente anterior a la de los Millennials o Nativos Digitales, podría desarrollar comportamientos más propios de esta última y que, igualmente, personas que por su edad podrían ser incluidas dentro de la generación de Millennials o nativos digitales, desarrollan comportamientos equiparables a los de generaciones anteriores como consecuencia de su educación, trayectoria vital o capacidad de acceso a la tecnología.

La combinación de los espacios físicos y los virtuales es otra de las características del comportamiento de esta generación. Como ya señaló, el paradigma tecnoeconómico ha comenzado a dar paso a una nueva representación en la que los espacios virtuales en modo alguno están sustituyendo a los espacios físicos, pues las técnicas virtuales permiten sumergirse en la imagen, actuar y trabajar en un universo tridimensional. Bajo este punto de vista, no podría hablarse de que lo virtual y lo físico sean dos realidades incompatibles, pues contrariamente a lo interesadamente sostenido por algunos, los espacios virtuales no están pensados para un uso individual sino para la sociabilización. Es un hecho social que la llegada y popularización de la Web 2.0 ha acentuado este proceso en su uso por los ciudadanos.

Es en este punto es donde se visualiza una de las principales diferencias con respecto al impacto de la tecnología en la vida de las distintas generaciones, pues para los nativos digitales no es posible separar la tecnología de su vida personal, ya que consideran al mundo virtual como parte esencial de su entorno natural y, por ello, tan real como el mundo físico. Asistimos, por tanto, a un acelerado proceso de transformación en el que estos jóvenes están llevando a cabo importantes cambios cognitivos, sociales y culturales que, de forma inevitable, van a verse reflejados en aspectos relevantes de nuestra sociedad como la educación, la economía y el empleo, la cultura, la política y la información y la comunicación. En el debate académico, se valora como el impacto que ha generado la Revolución tecnológica en el desarrollo en general y en especial en el escenario académico cubano, donde el rol del Estado no puede estar ausente, no sólo porque sigue siendo un actor de la vida política, económica y social, sino porque incide en el cambio tecnológico como un paradigma social al ser el encargado de dotar a los escenarios donde se educa de estas tecnologías. La difusión de la tecnología amplifica infinitamente su poder al apropiársela y redefinirla sus usuarios. Las Tics no son sólo herramientas que aplicar, sino procesos que desarrollar, y en 
este escenario es de suma importancia ponderar su implementación en las formas de gestión (CASTELLS, 1999, p. 48).

Soler, Antúnez, Mercado y Ramírez (2015), significaron que en la actualidad las universidades deben promover experiencias innovadoras en las actividades de postgrados, apoyadas en el uso de las tecnologías, contrarias al modelo tradicional que por muchos siglos ha sido implementado en las aulas de manera presencial en Cuba. Permite demostrar el énfasis que debe hacerse en la docencia, en los cambios de estrategias didácticas de los profesores, en los sistemas de comunicación y distribución de los materiales de aprendizaje, en lugar de enfatizar la disponibilidad y las potencialidades de las tecnologías.

En consecuencia, se justiprecia que todos estos análisis en torno a los criterios acerca de la definición de que es la Infotecnología y para que se implementa, se considera como es que, a principios del siglo $\mathrm{XXI}$, se afirmara:

El buen manejo de los computadores y de la Internet es una de las habilidades que deben caracterizar al ciudadano competente en el siglo XXI. Lograr entonces que al terminar su etapa escolar los jóvenes dominen las herramientas básicas de las Tics es un objetivo importante del plan curricular de cualquier institución educativa (PIEDRAHITA PLATA, 2003, p. 27).

Por lo que, en consecuencia, con todos los análisis realizados en torno a los criterios acerca de la infotecnologías, se valora como es a principios del siglo XXI donde se afirmara que: "El buen manejo de los computadores y de la Internet es una de las habilidades que deben caracterizar al ciudadano competente en el siglo XXI". Es por ello, que para una adecuada preparación con esta herramienta a la sociedad civil, dependerá de resultados exitosos en el cumplimiento del principio de acceso a la información ambiental, donde aparecen vinculadas en su aplicación y utilidad a las ciencias contables, a la protección ambiental, al desarrollo productivo, a la implementación de tecnologías limpias, en el desarrollo del comercio interno y foráneo, entre otras disciplinas afines aportando valores agregados en el mundo de la competencia empresarial "marketing empresarial", ejemplo de ello sería completado si son realizadas las audiencias ambientales con los ciudadanos, a través de la adecuada información sobre los sistemas de etiquetado ambiental, entre los instrumentos de mercado de carácter cooperador que hoy son socializados e implementados.

Donde, en la llamada "sociedad de la información y el conocimiento", ante la generación de un volumen insospechado de información en el mundo empresarial constituye un reto para los profesionales y para la sociedad en general. Al resultar imprescindible la formación continua de estos para salvar la brecha de la infoxicación, discernir entre el enorme volumen de información existente y la de alta calidad; es así que también constituye una prioridad desarrollar en el profesional capacidades que le permitan guiarse a través del intrincado laberinto de información y obtener aquella que precisa para desplegar con rigor su actividad profesional dentro de las formas de gestión donde tenga relación jurídica activa o pasiva.

No obstante, se aprecia que con el uso del e-Learning, como la plataforma digital pública, permitirá de manera rápida y eficaz adquirir las habilidades y conocimientos básicos sobre la formación ambiental y así de manera universal informar a los ciudadanos de los problemas ambientales mundiales y de la región donde residen; la que se combina con los últimos avances de la tecnología multimedia para activar los sentidos de los usuarios que la utilizan para el desarrollo del conocimiento básico en la problemática ambiental y de cómo incidir en el cambio y en la percepción del riesgo, todo un gran reto al no estar concebidas como un derecho cívico en cada Estado, por los altos costes que genera su implementación.

Hoy el tener acceso a dispositivos digitales como la Tablet, un Móvil Androide, una Laptop, y todos los que estarán por venir con el desarrollo acelerado de la Cibernética y or supuesto conocer su adecuada manipulación y uso, conllevara a acceder a la nube de información, permitirá lograr la adecuada formación ambiental del ciudadano para incidir en su conciencia de preservar el medio ambiente para las futuras generaciones y alcanzar el desarrollo sostenible en las formas de gestión con relación a las metas ambientales diseñadas por cada objetivo propuesto por la Administración Pública dentro de las formas de gestión. 


\section{Las metas ambientales en las formas de gestión en Cuba. ¿Qué objetivos persiguen?}

La problemática ambiental del primer decenio del siglo XXI, no podría ser analizada ni entendida sin tener en cuenta la perspectiva global que explicase sus raíces originarias como una consecuencia de los múltiples factores como son los efectos a partir de la Revolución Industrial del pasado siglo XX, y de las nuevas tecnologías que le han seguido las que no hay tenido en cuenta el equilibrio entre estas y la protección de la naturaleza, entre ellas: el aumento de las temperaturas, el agujero en la capa de ozono, la desertificación, la acumulación de residuos radiactivos, la extensión de enfermedades como el cáncer, la malaria, la insalubridad del agua dulce, la inseguridad alimentaria, la contaminación urbana y el agotamiento de los recursos renovables y no renovables, el calentamiento global, entre otras.

Dentro del desarrollo del Derecho Ambiental, se precia como priman principios rectores que establecen la actividad derivada de la protección del medio ambiente, a partir de que el principio del derecho a un medio ambiente sano, reconocido en la Declaración de las Naciones Unidas de 1948, con su consagración del derecho de toda persona a un nivel de vida adecuado, que le asegure a él y a su familia, salud y bienestar, como una primera base en la que pudiera asentarse este derecho al medio ambiente, los que no son del todo conocidos por los ciudadanos y en qué momento estos deberán ser observados.

Se plantea que la primera mención expresa de ese principio aparece en el Pacto Internacional de los Derechos Económicos, Sociales y Culturales de 1966, donde se establece directamente el carácter del ambiente como un requisito indispensable para el desarrollo de la persona. En este íter, el principio de Prevención, en la Declaración de Río señala: La prioridad de la prevención mediante la adopción de medidas sobre una base científica y con los estudios técnicos y socioeconómicos que correspondan. Asumir la prevención a manera de herramienta tiene otros efectos más específicos en las políticas ambientales, se cita el concepto de producción más limpia como una de las consecuencias de la instrumentación efectiva del principio de prevención.

El principio Precautorio, refleja en el numeral 15 de la Declaración de Río, y plantea: con el fin de proteger el medio ambiente, los estados deberán aplicar ampliamente el criterio de precaución conforme a sus capacidades. Cuando haya peligro de daño grave o irreversible, la falta de certeza científica absoluta no deberá utilizarse como razón para postergar la adopción de medidas eficaces en función de los costos para impedir la degradación del medio ambiente. El principio de responsabilidad, se presenta bajo dos formas: como responsabilidad frente a daños causados por efectos de la contaminación ambiental a personas físicas y sus bienes y a personas jurídicas y sus bienes de parte de personas similares, y, como responsabilidad del Estado por contaminación ambiental que afecte tal medio ambiente de otro Estado, produciéndose un daño significativo. En la primera existen varias convenciones que se refieren a la responsabilidad civil por daños ocasionados a personas o bienes de terceros; y con respecto a la segunda la responsabilidad estatal aparece expresamente establecida sólo en determinados Tratados desde el Derecho Internacional.

En esta misma línea histórico jurídica, se valora como la Cultura de la Información, reconocida como el conjunto de hábitos, habilidades y valores que una persona emplea para "reconocer cuando es necesaria la información y poder localizar, acceder, organizar, evaluar y usar con efectividad la información que necesita", la que puede incidir en la formación de la cultura ambiental de manera adecuada dentro de los objetivos ambientales que se tracen las formas de gestión para el logro de sus metas ambientales.

El concepto de Cultura de la Información se constata como se vincula con el uso ético de la información, aspecto muy importante en nuestro medio, y el cumplimiento de las normas que la rigen, y en especial con la formación de una cultura de intercambio y producción de conocimiento.

Es así, que en la segunda década del siglo XXI, se analiza como uno de los principios que viene revolucionando el actuar de la Administración Pública y al Derecho Administrativo es el de participación pública, renovándose y replanteándose, este señala: “[...] el principio de la participación pública exige la 
participación democrática en los procedimientos decisorios sobre materias de repercusión ambiental [...]". Toda vez que desde el 1982, la Carta Mundial de la Naturaleza afirmaba en el punto 23 de la misma que:

[...] toda persona, de conformidad con la legislación nacional, tendrá la oportunidad de participar, individual o colectivamente, en el proceso de preparación de las decisiones que conciernan directamente a su medio ambiente y, cuando éste haya sido objeto de daño o deterioro, podrá ejercer los recursos necesarios para obtener una indemnización [...].

Por estas razones, se pondera que las metas ambientales dentro de las formas de gestión cubanas deberán perseguir la formación de la cultura ambiental del ciudadano en atención a los elementos antes señalados de manera adecuada, ello permitiría y contribuiría a lograr alcanzar los objetivos propuestos, y con ello contribuir entre otros logros, obtener dentro de las formas de gestión los reconocimientos honoríficos en materia ambiental concedidos por el Ministerio de Ciencias, Tecnología y Medio Ambiente, luego de ser objeto de la inspección ambiental, o de una auditoría ambiental por parte de la Contraloría General de la República, certificándola como empresa o industria limpia, esta última actividad de control le aportaría valores añadidos a las formas de gestión, lanzándola con mejores competencias al mercado interno y al foráneo. Inobjetablemente se estaría ponderando la protección adecuada del bien público ambiente en pos de alcanzar el desarrollo sostenible como así se dispone en la carta magna cubana.

Es también un hecho que, el siglo XXI presenta al mundo imperiosos desafíos en relación con el medio ambiente, como fruto del sorprendente avance tecnológico e industrial de las últimas décadas del pasado siglo, hasta convertirse en lo que señalan algunos autores como la crisis ecológica, como hechos que no han tenido toda la difusión básica de los ciudadanos de manera adecuada, del que la nación cubana no escapa. En consecuencia con lo antes dicho, el Derecho Ambiental como ciencia social, se justiprecia como se ha constituido en la disciplina destinada a proveer los instrumentos jurídicos para afrontar las exigencias de protección del bien público ambiente, ha tenido un reconocimiento para su protección en América Latina desde los textos constitucionales, siendo las naciones de Bolivia y Ecuador fieles exponentes del Pluralismo jurídico, la primera por considerar como sujeto a la madre tierra (Pacha Mama) y la otra el buen vivir (Suma Kwasay). (DÍAZ OCAMPO; ANTÚNEZ SÁNCHEZ, 2017).

Por ello, no ha quedado detrás la preocupación de los empresarios ante la problemática ambiental, declarada en los cónclaves universales realizados desde Rio de Janeiro en 1992 hasta Paris en el 2015, han estado enfocadas a ponderar propuestas para disminuir la contaminación generada por las industrias en sus procesos industriales; empero, las respuestas dadas por los líderes de los Estados, aún no han sido adecuadas en su mayoría al no existir consenso en adoptar acuerdos para mitigar la contaminación que afecta el equilibrio natural del planeta, lo que ha incidido en el calentamiento global, generando efectos negativos al medio ambiente, los que inciden en la salud de los ciudadanos, en la biodiversidad, entre otros.

La situación antes apuntada, lleva a analizar la conexión de los problemas ambientales y la práctica del control administrativo a través de instrumentos de gestión ambiental a través de la función inspectiva y la auditoría como actividad de control para evaluar la legalidad, apreciada con los orígenes del ius ambientalismo como parte de la agenda pública internacional a ejecutarse por los servidores públicos de la Administración Pública en los Estados, como es el caso de la nación cubana con la implementación de las herramientas de gestión con la Estrategia Ambiental Nacional, el Plan de lucha contra la contaminación, regulados en la Ley $n$. 81 de 1997, donde se declaran los problemas ambientales que afectan al medio ambiente y se destinan políticas para su mitigación, protección y preservación.

Por la transversalidad de la cuestión ambientalista, se reconoce su vínculo con otras ramas de las ciencias jurídicas y con otras ciencias como la ecología, la contabilidad, la sociología, la psicología ambiental, la historia, las ciencias ambientales, la biotecnología ambiental, las TIC's, la geografía, la geología y la economía, entre otras ramas y saberes, que permiten de manera integrada dar soluciones a los daños generados, del cual se valora que los ciudadanos dentro de las formas de gestión deben 
empoderarse de estos conocimientos elementales por sus elementos técnicos, que le permitan con su actuar incidir en su mitigación y solución.

Pero esta aplicación de conocimientos y respuestas, se valora que necesita de su empoderamiento cognoscitivo por parte de los ciudadanos, para que contribuyan a la protección del medio ambiente de manera adecuada como un bien público, que les permita actuar ante la antijuricidad cometida en las formas de gestión de la nación cubana. No puede olvidarse que, a partir del primer compromiso internacional, con la decisión de las Naciones Unidas de declarar al año 1970, como el "Año internacional del medio ambiente" esta protección se ha exteriorizado en tres dimensiones: la económica, la social y la ambiental a partir del desarrollo industrial equilibrado para generar recursos, empleos y promover la educación ambiental como bases del bienestar social y calidad de vida, para lograr el principio del desarrollo sostenible, contextualizado con la Responsabilidad Social Empresarial (RSE).

En este plano socio jurídico, no debe ser olvidado que acciones ejecuta el CITMA, como organismo de la Administración Pública, quien conformó el Plan de Lucha contra la contaminación destinado a las formas de gestión, en pos de que sean generadas acciones que tributen a mitigar los daños al ambiente. Los autores consideran que es esta una de las causas por las que el Derecho a la información deba ser ponderado por todos los Estados, que permita cumplir el principio constitucional de deber-derecho ante los episodios negativos de contaminación por la extracción de minerales, de petróleo, devastación de los bosques, y contaminación de las aguas, al no observarse de manera adecuada los principios de precaución, prevención, legalidad y el de responsabilidad que desde esta rama del Derecho deben ser tenidos en cuenta para lograr una adecuada conciencia ambiental, en pos de la protección del bien público ambiente.

Hoy el problema del cambio climático, por su importancia es apreciada en la comprobación de las políticas del Estado, donde se incorpora la dimensión ambiental, ponderada en el siglo XXI ante las consecuencias negativas generadas, analizadas en el último conclave internacional en Paris 2015 como se reseñó, para ello en Cuba se ha aprobado el Proyecto Vida, el que traza estrategias a cumplir por las formas de gestión y los ciudadanos en pos de la preservación del medio ambiente con políticas publicas dirigidas a este fin.

Esta protección al bien público ambiente, se articula a partir del reparto de las atribuciones y competencias dentro del ordenamiento jurídico, las que parten desde la Constitución, a partir de los principios de organización y actividad de los órganos estatales establecidos en el artículo 68, y en virtud de estos se integran y desarrollan sus acciones sobre la base de la democracia socialista, en las tareas de su competencia, la obligatoriedad de las disposiciones de los órganos superiores para los inferiores, la subordinación y la rendición de cuentas de estos últimos a los superiores, lo que garantiza la articulación y el control de la política ambiental en las formas de gestión. Es por ello, que el Estado cubano estableció un modelo institucional nuevo, sustentado en una instancia coordinadora de gestión ambiental, coexistiendo con la distribución de competencias previas respecto a los recursos naturales y a los sub sistemas ambientales. Por ello, la función pública ambiental cubana, ejecutada por los servidores públicos, se complementa con la declaración de soberanía estatal sobre los recursos naturales y el medio ambiente, proclamada en el artículo 11, del texto constitucional en unión a los artículos 10 y 27 . Estos artículos conforman las bases constitucionales fundamentales que validan la práctica del control público para proteger el ambiente con observancia de la legalidad. Reproducen el principio del desarrollo económico y social sostenible contenido en los principios 3 y 4 de la Declaración de Río de Janeiro de 1992, como bien público tutelado.

\subsection{Las metas ambientales en las formas de gestión}

A partir que el derecho a la información se ha reconocido en los textos constitucionales de la región y en especial en la nación cubana, los autores aprecian que es este un derecho doble: el derecho a 
comunicar y el derecho a recibir información. Donde sí se analiza hay una vertiente activa y otra pasiva. Se ha señalado por que la fortaleza del Derecho en su dimensión objetiva es la de trasmitir información, en el caso sería la ambiental. El derecho a la información pública se ha convertido en un derecho humano esencial para el desarrollo de la democracia.

En esta "Sociedad de la Información y el Conocimiento" huelga señalar su importancia tanto en su dimensión individual como en la colectiva, así como la relevancia que tiene tal derecho para hacer accesible y posible el disfrute de otros derechos humanos. Es una herramienta clave para que la ciudadanía ejerza su protagonismo cívico en el control de la cosa pública o del manejo que de esta hacen sus representantes. El desarrollo de la gestión ambiental, es un proceso que ha estado marcado por un grupo de condiciones naturales, históricas, políticas y económicas específicas para Cuba, han ejercido una influencia en el devenir y, sobre todo, en la conceptualización y práctica de la gestión ambiental integral. A partir de lo que se establece en la Ley n. 81 de 1997, se define que la gestión ambiental aplica la política ambiental mediante un enfoque multidisciplinario, ha tenido en cuenta el acervo cultural, la experiencia nacional acumulada y la participación ciudadana para incidir en la protección ambiental.

En su evolución, la protección al medio ambiente durante más de cincuenta años, ha sido en constante perfeccionamiento y maduración, con logros ambientales relacionados con el desarrollo de la educación, la eliminación de la pobreza extrema, el acceso al empleo, la salud, la equidad. Un ejemplo de ello ha sido, la creación y el desarrollo de instituciones ambientales de investigación, lo que ha permitido el constante conocimiento de los recursos naturales, vinculadas al trabajo decente, en atención al carácter humanista de este proceso, en este escenario aparece la Empresa de Alta Tecnología, la que por sus características es respetuosa con el ambiente, no así para el resto de las formas de gestión estatal, las que por su heterogeneidad y obsolescencia tecnológica deberán ir cambiando sus patrones de conducta.

Se preconiza, que dentro de la gestión ambiental se deberá ponderar los principios del Derecho Ambiental como el de prevención, precaución, responsabilidad, legalidad, cooperación, información, quien contamina paga y el de desarrollo sostenible, establecidos a partir del texto constitucional en el artículo 27, y desarrollado en la Ley n. 81 de 1997. Los que no se observan de manera adecuada por las formas de gestión, generando impacto negativo en la industria por la contaminación y del sector secundario al no ponderar los Objetivos del Desarrollo del Milenio (ODM), los que centran en su potencial valor añadido para contribuir al desarrollo humano y social, que como sujeto activo le corresponde su implementación y control administrativo. "Los ODM aún son realizables. La pregunta crítica hoy es cómo conseguir que el camino hacia el cambio progrese a partir de ahora mucho más rápidamente." En este sentido los ODM incluyen un objetivo específico para las asociaciones globales. En el objetivo 8: "[...] Desarrollar una asociación global para el desarrollo", se da un llamamiento específico para que "el sector privado y la industria se unan a otros actores sociales como las IES en la lucha a favor del desarrollo global humano y social [...]".

En Cuba, el control ambiental se ha ejecutado de manera mayoritaria por la función inspectiva a través de los procedimiento establecidos por la Resolución n. 103 del 2008 del CITMA, en relación a la Ley n. 81 de 1997, en ella se evalúan las formas de gestión, a las que les acredita la condición ambiental y le concede la etiqueta-certificación a los productos y servicios como sujetos estratégicos; se corrobora como en la nación el sector turístico es a quien ha sido objeto de este control para su acreditación, sin desdeñar el sector productivo que despunta en este sentido. Aunque, la inspección ambiental se constriñe al control público de los ecosistemas de interés de la nación. Ahora bien, se considera que la misma se complementa con la inspección sanitaria estatal, ejecutada por el Ministerio de Salud Pública, la cual evalúa el sector estatal y el privado, establecida en la Ley n. 41 de 1983, por parte de los inspectores sanitarios.

Se valora, como el marco regulatorio complementario a la Ley n. 81 de 1997, aún no garantiza la adopción de enfoques preventivos adecuados, aparejados a la indisciplina tecnológica que impera en la mayoría de los sujetos de gestión; al no valorarse de manera adecuada los avances científicos, denominada desde la doctrina como -cláusula de progreso científico-, aspectos que deberán ser tenidos en cuenta en la futura actualización normativa y la del Derecho de la Empresa al realizarse la contratación mercantil. 
Por estar está condicionada al principio de seguridad jurídica que permita garantizar el equilibrio adecuado entre la mejor tecnología a implementar y los costes económicos que puedan acarrearse a futuro. Razón por la cual se considera potenciar a través del uso de las infotecnologías estos valores ambientales, para que contribuyan a mitigar y dar una protección adecuada al bien público ambiente.

En las formas de gestión, se valora como aún no se pondera de manera adecuada dentro de los análisis vinculados al Informe Cosso II, la protección del medio ambiente, la implementación de las etiquetas ecológicas, los programas ambientales, los programas de certificación y de adquisición de productos ecológicos, los que tienen como objetivo fortalecer los mercados con orientación ecológica, la disminución de los riesgos ambientales, entre otros. Para ello, la incidencia el ecoetiquetado, está concebido como instrumento de mercado desde la doctrina del Derecho Administrativo Ambiental, se establece como la tarjeta de presentación del producto y su garantía de calidad. Le corresponderá, por su relación con el Derecho de Propiedad Industrial, que la información sea clara y legible, prohibitiva de publicidad falsa o engañosa, y del logotipo que la identifique. Permitirá, por su importancia, fomentar la Empresa Responsable con el Ambiente en la nación cubana, ante una cultura empresarial caracterizada en su mayoría por la no adecuada utilización de los instrumentos financieros, no evaluadora de la dinámica del mercado, donde no se analizan los niveles de información de cómo opera la competencia empresarial con el empleo de técnicas comerciales, las tecnologías y la innovación para su desarrollo futuro, a tono con la norma ISO 26000 y el Libro Verde implementado en la Unión Europea, muy ligada al autocontrol empresarial con el Cosso II, obtener una empresa limpia, donde el uso de las infotecnologías podrá jugar un rol principal en la socialización de conocimientos.

Desde la Ciencia de la Administración, se aprecia como también se le podría incorporar el modelo de la Triple Hélice, que permita contextualizar y socializar el principio de participación, principio este estructural desde el Derecho Administrativo Ambiental, conectado al principio del desarrollo sostenible como el mega principio del Derecho Ambiental, para alcanzar de manera adecuada con la implementación de las Tics la cultura ambiental como objetivo dentro de las metas ambientales en las formas de gestión cubanas, como un valor.

Es también un hecho que, el papel de la ciencia cubana está en los tres documentos rectores del modelo económico y social cubano a aprobarse en este 2017, es decir, en la conceptualización del modelo económico y social, en el Plan nacional de desarrollo hasta el 2030 y en los Lineamientos de la Política Económica, se valora que la nación va en la dirección correcta, pero no al ritmo que ya debería haberse avanzado, por lo que aún quedan muchas ideas que no han sido institucionalizadas de forma adecuada, necesitadas de una construcción desde las Ciencias Sociales y afines, de las que el Derecho no escapa, para su regulación en cuerpos jurídicos dentro del ordenamiento jurídico, donde la protección del bien público ambiente está señalado como una necesidad, entonces socializarlas aún más con el uso de las infotecnologías continua siendo un reto, el que puede ser logrado.

\section{Conclusiones}

Las infotecnologías, a partir de estar concebidas como el conjunto de conocimientos y habilidades prácticas de los individuos, ser las herramientas indispensables para mantener las relaciones exitosas con el nuevo entorno y con otros individuos, puede ser aprovechada para la formación ambiental adecuada en las formas de gestión del sector empresarial, ello contribuirá a lograr las metas ambientales propuestas como un valor añadido.

La problemática ambiental cubana en las formas de gestión, deberá ponderar la inclusión de las herramientas que aportan las Tics, las que tributarán a formar valores ambientales en los ciudadanos en relación a apropiarse de información y conocimientos de cómo opera la competencia empresarial con el empleo de técnicas comerciales, las tecnologías y la innovación para su desarrollo futuro, a tono con la 
norma ISO 26000 y el Libro Verde, ligada al autocontrol empresarial con el Cosso II, en pos de desarrollar empresa limpias en la protección del medio ambiente.

En Cuba, es una necesidad alcanzar una cultura ambiental adecuada dentro de las formas de gestión estatales y privadas, su difusión a través de las infotecnologías podrá contribuir a que sean mucho más competitivas en el mercado interno y el foráneo, donde sea una meta ambiental alcanzar la empresa responsable con sus productos y servicios certificados con la etiqueta ambiental, tal y como se viene reconociendo en los cambios en el nuevo modelo económico y social.

Una de las metas ambientales futuras de la nación como paradigma de la Administración Pública, es lograr una cultura ambiental, que pondere la protección del bien público ambiente. Con ello se tributará a alcanzar el desarrollo sostenible, y el uso y aplicación de las herramientas digitales como las infotecnologías pueden lograr a alcanzar esta propuesta.

\section{Referencias}

MERCHÁN, E.; MERO, Karina; ANTÚNEZ, G. Impacto de las tecnologías de la información y las comunicaciones: Una mirada en actividades formativas. Revista electrónica de Veterinaria, España, v. 16, n. 7, p. 1-10, 2014. Disponível em: <http://www.veterinaria.org/revistas/redvet/n070715/071503.pdf>. Acesso em: 12 mar. 2017.

ANTÚNEZ SÁNCHEZ, Guillermo et al. Curso virtual de redacción científica e infotecnología sobre la plataforma Moodle: resultados y experiencias. Píxel-Bit. Revista de Medios y Educación, España, n. 41, p. 173-183, julio 2012. Disponível em: <hhttp://acdc.sav.us.es/ojs/index.php/pixelbit/article/ view/403/141>. Acesso em: 12 mar. 2017.

SOLER, Yolanda et al. Curso de infotecnología y redacción científica: El B-learning para profesionales de las Ciencias Agropecuarias. Revista electrónica de Veterinaria, España, v. 13, n. 11, 2012. Disponível em: <http://www.veterinaria.org/revistas/redvet/n111112/111208.pdf>. Acesso em: 12 mar. 2017.

FONSECA, Raquel Zamora; IGLESIAS, Carlos Cañedo; DÍAZ, Magdiel Chaviano. La gestión didáctica en la infotecnología. Una experiencia en la Universidad de Cienfuegos. Biblios, España, n. 31, p. 1-8, abr./jun. 2008. Disponível em: <http://file:///C:/Users/730546414/Downloads/Dialnet-LaGestionDidacticaE nLalnfotecnologia-2663200.pdf>. Acesso em: 12 mar. 2017.

CARNEIRO, Roberto; TOSCANO, Juan Carlos; DÍAZ, Tamara (Coord.). Los desafíos de las Tics para el cambio educativo. España: Fundación Santanilla, 2008.

ÁLVAREZ HINCAPIÉ, C. Sistemas de certificación ambiental para la extensión tecnológica, la competitividad empresarial. Revista P+L, Colombia, v. 3, n. 2, p. 61-87, jul./dic. 2008.

ANTÚNEZ SÁNCHEZ, A.; RONDÓN VALDÉS, J. La formación de habilidades en los estudiantes de la carrera de derecho. Mapas conceptuales. Aprendizaje cooperativo. Un recurso indispensable en la formación universitaria. España: Universidad de Valencia, 2016.

ANTÚNEZ SÁNCHEZ, A. El uso de las infotecnologías para desarrollar el capital humano. De Jure revista jurídica, São Paulo, v. 15, n.7, p. 222-238, 2016.

ANTÚNEZ SÁNCHEZ, A. El modelo de la triple hélice y el desarrollo sostenible. Argentina: Urbe et lus, 2017.

ANTÚNEZ SÁNCHEZ, Alcides; OCAMPO, Eduardo Díaz. La responsabilidad socil empresarial. La empresa responsable con el ambiente. TJDFT, Brasília, ano 105, v. 970, p. 211-240, 2016.

ANTÚNEZ SÁNCHEZ, A. La responsabilidad social empresarial. Revista internacional Legis, Colombia, Foro de derecho mercantil, n. 51, p. 49-75, abr./jun. 2016.

ANTÚNEZ SÁNCHEZ, A. La empresa de alta tecnología. Revista Fórum Administrativo, Belo Horizonte, ano 16, n. 189, nov. 2016. 
AGIRREAZKUENAGA, I.; CHINCHILLA, C. El uso de medios electrónicos informáticos y telemáticos en el ámbito de las Administraciones Públicas. Revista Española de Derecho Administrativo, Madrid, $\mathrm{n}$. 109, p. 35-60, 2001.

AYES AMELLIER, G. Medio Ambiente, impacto y desarrollo. La Habana: Editorial Científico-Técnica, 2003.

BATES, T. Cómo gestionar el cambio tecnológico. Barcelona: Gedisa, 2001.

BUSTOS, A.; COLL, C. Los entornos virtuales como espacios de enseñanza y aprendizaje. Una perspectiva psicoeducativa para su caracterización y análisis. Mexicana de Investigación Educativa, México, v. 15, n. 44, p. 163-84, 2010. Disponível em: <http://scielo.unam.mx/pdf/rmie/v15n44/v15n44a9. pdf>. Acesso em: 10 ago. 2013.

CABERO ALMENARA, J.; LLORENTE, M. C. Las plataformas virtuales en el ámbito de la teleformación. Alternativas de Educación y Comunicación. Argentina. 2005. Disponível em: <http:// www.e-alternativas.edu.ar/>. Acesso em: 10 ago. 2013.

CABERO ALMENARA, J. Comunidades virtuales para el aprendizaje. Su utilización en la enseñanza. EDUTEC -Revista Electrónica de Tecnología Educativa, España n. 20, p. 1-20, 2006. Disponível em: <http://www.edutec.es/revista/index.php/edutec-e/article/viewFile/510/244>. Acesso em: 10 ago. 2013.

CABERO ALMENARA, J.; LLORENTE, M.C. La formación semipresencial a través de redes telemáticas (blended learning). Da Vinci: Mataró, 2008.

CABERO ALMENARA, J. Los retos de la integración de las TICs en los procesos educativos. Límites y posibilidades. Perspectiva Educacional, Chile, v. 49, n. 1, p. 32-61, 2010.

CABERO ALMENARA, J. Nuevos escenarios tecnológicos para innovar en la educación. En: SEMINARIO IBEROAMERICANO DE INNOVACIÓN DOCENTE., I. España, Universidad Pablo de Olavide, Sevilla, 20 y 21 de noviembre 2014. Disponível em: < https://documentop.com/nuevosescenarios-tecnologicos-para-innovar-en-la-educacion-upo_5a011ce01723dd1144bd213e.html>. Acesso em: 10 ago. 2015.

CABERO ALMENARA, J. Bases pedagógicas del E-learning. España: Universidad y Sociedad del Conocimiento, 2006.

CABERO ALMENARA, J. Las necesidades de las TIC en el ámbito educativo: oportunidades, riesgos y necesidades. Tecnología y Comunicación Educativa, España, ano 21, n. 45, p. 5-19, jul./dic. 2007

CASTELLS, Manuel. La dimensión cultural de Internet. España: Universitat Oberta de Catalunya, n. 36, p. 7-10, abr. 2003. Disponível em: <http://www.uoc.edu/culturaxxi/esp/a>. Acesso em: 23 mar. 2017.

CAMPIÓN, S.; NAVARIDAS NALDA, F. La web 2.0 en escena. Revista Pixel-Bit, España, Universidad de Sevilla, n. 41, p. 19-30, julio 2012.

CIRILO AGUILAR, B. Educación virtual: fractura de un paradigma. Revista Dimensión Empresarial, México, n.1, p. 74-79, 2011.

CEBRIÁN, Mariano. La web 2.0 como red social de comunicación e información. Estudios sobre el Mensaje Periodístico, Madrid, v. 14, p. 345-361, 2008.

DE PABLOS PONS, J.; CORTÉS, R Jiménez. Buenas prácticas con TIC apoyadas en las Políticas Educativas: claves conceptuales y derivaciones para la formación en competencias ECTS. Revista Latinoamericana de Tecnología Educativa, España, Sevilla, v. 6, n. 2, p. 15-28, 2007.

DELPIAZZO, C.; VIEGA, M. Lecciones de derecho telemático. Montevideo: FCU, 2009.

DUART, J. M.; SANGRÀ, A. Aprender en la virtualidad. Barcelona: Gedisa, 2000. t. I-II.

DONINI, A. Desafíos de la sociedad del conocimiento a las políticas académicas y científicas: La gestión universitaria en el siglo XXI. Buenos Aires: Universidad de Belgrano, 2003. 
DÍAZ OCAMPO, E.; ANTÚNEZ SÁNCHEZ, A. La justicia indígena y el pluralismo jurídico en Ecuador. El constitucionalismo en América Latina. Revista Derecho y Cambio Social, Perú, n. 44, p. 1-38, 2016. Disponível em: <http:// file:///C:/Users/730546414/Downloads/Dialnet-LaJusticialndigenaYEIPluralismoJur idicoEnEcuador-5456253.pdf>. Acesso em: 23 mar. 2017.

FANDOS, M. Formación basada en las tecnologías de la información y comunicación: análisis didáctico del proceso de enseñanza-aprendizaje. Tesis Doctoral. Tarragona: Facultad de Ciencias de la Educación, 2003.

GARRIDO, Manuel Fandos. Formación basada en las tecnologías de la información y comunicación: análisis didáctico del proceso de enseñanza-aprendizaje. Tesis Doctoral. Tarragona: Facultad de Ciencias de la Educación, 2003. 341 f.

FERRÁS SEXTO, C. Construyendo la sociedad digital: un desafío para las ciencias sociales. Revista Electrónica Teoría de la Educación, España, n. 2, p.5-7, 2009.

FERNÁNDEZ RAMOS, S. La inspección ambiental. Revista del Medio Ambiente y la Administración Local, España, p. 1-26, 2012.

FERNÁNDEZ DE GATTA SÁNCHEZ, D. Las auditorías ambientales. España: Tirant lo Blanch, 2008.

FLORES, O. TIC y docencia universitaria: ¿cambian las metodologías docentes según el grado de presencialidad de las asignaturas? El caso de la Universidad de Lleida. Pixel-Bit Revista de Medios y Educación, España, n. 41, p. 63-76, 2012. Disponível em: <http://acdc.sav.us.es/pixelbit/images/stories/ p41/05.pdf>. Acesso em: 23 mar. 2017.

ECHEVERRÍA, J. Educación y Tecnología Telemáticas. Revista Iberoamericana de Educación, España, n. 24, p. 17-36, 2000.

ETZKOWITZ, H.; LEYDESDORFF, L. University and the global knowledge economy: a triple helix of university- industry- government relations. London: Pinter Publishers, 1997.

GARCÍA FERNÁNDEZ, J.; REY SANTOS, O. Foros de negociación e instrumentos jurídicos internacionales en materia de medio ambiente y desarrollo sostenible. La Habana: Acuario, 2005.

GROSSMAN, L. Time's person of the year: you. 2006. Disponível em: <http://www.time.com/time/ magazine/ article/0,9171,1569514,00.html>. Acesso em: 24 ago. 2016.

LÓPEZ DE LA MADRID, M.C. Uso de las TIC en la educación superior de México: un estudio de caso. Apertura, Guadalajara, v. 7, n. 7, p. 63-81, 2006.

LEE, F.; CARBONELL, S.; TORICELLA, R. Infotecnología: la cultura informacional para el trabajo en la Web. Habana: Editorial Universitaria, 2008. Disponível em: <http://libros.metabiblioteca.org/ bitstream/001/486/1/informacion_en_web.pdf> Acesso em: 23 mar. 2017.

LILIANA CORIA, S. ¿Nuevo paradigma? $4^{\text {ta }}$ Revolución industrial. Revista Iberoamericana de Derecho Ambiental y Recursos Naturales, Argentina, IJ-CCLXIII-567, n. 23, marzo, 2017.

LOZANO CUTANDA, B. El futuro de la flor europea: la nueva etiqueta ecológica de la UE. Revista Catalana de Derecho Ambiental, España, v. 1, n. 1, p. 1-16, 2010.

LLANEZA, P. Derechos fundamentales e Internet. Cuadernos de comunicación e innovación, Madrid, n. 85, p. 54-57, 2010.

GONZÁLEZ DE LA FE, T. El modelo de triple hélice de relaciones universidad, industria y gobierno: un análisis crítico. Arbor Ciencia, Pensamiento y Cultura, España, v. 185, n. 378, p.739-755, 2009.

MARTÍN MATEO, R. El etiquetado ecológico, nuevo instrumento para la tutela ambiental. España: Trivium, 1994.

MONEREO, C. Internet y competencias básicas: aprender a colaborar, a comunicarse, a participar, a aprender. Barcelona: Graó, 2005.

MORA RUÍZ, M. La gestión ambiental compartida: función pública y mercado. España: Lex Nova, 2012. 
NOVAK, J. D. Aprendiendo a aprender. Barcelona: Martínez Roca, 1988.

NOGUEIRA LÓPEZ, A. Ecoauditorías, intervención pública ambiental y autocontrol empresarial. España: Marcial Pons, 2010.

NIETO GÖLLER, R. Educación virtual o virtualidad de la educación. Historia de la Educación Latinoamericana, Argentina, n. 19, p. 137-150, 2012.

NIETO GÖLLER, R. Educación virtual o virtualidad de la educación. Revista Historia de la Educación Latinoamericana, Boyacá, Colombia, v. 14, n. 19, p. 137-150, 2012.

PAREDES LABRAC, J. Usos educativos de la telemática. Madrid: Universidad Autónoma de Madrid, 2010.

PASTOR SÁNCHEZ, J. La gestión del conocimiento en las universidades: el nuevo documento electrónico de la tabla al hiperdocumento. Murcia: Universidad de Murcia, 1996.

PALOMAR OLMEDA, A. EI procedimiento administrativo electrónico. Curso sobre Administración electrónica en los Gobiernos y Administraciones locales en Huesca. España, 2009.

PÉREZ LUÑO, A. Internet y el Derecho. Revista Iberoamericana de Informática y Derecho, Mérida, v. 1, n.19-22, p. 721-734, 1998.

PÉREZ LUÑO, A. Manual de informática y derecho. Barcelona: Ariel, 1996.

PÉREZ LUÑO, A.¿Ciberciudadaní@ o Ciudadaní@.Com? Barcelona: Gedisa, 2004.

PIÑAR MAÑAS, J. L. Revolución tecnológica, derecho administrativo y administración pública: notas provisionales para una reflexión. Madrid: Civitas, 2007.

PÉREZ SERRANO, G.; SARRATE CAPDEVILA, M. L. Las Tics, promotoras de inclusión social. Revista española de Pedagogía, Madrid, n. 249, p. 237-254, 2011.

PLATERO ALCÓN, A. El derecho al olvido en internet. El fenómeno de los motores de búsqueda. Opinión Jurídica, Colombia, n. 29, p. 243-260, 2015.

PIGRETI, E. Ambiente y sociedad - El bien común planetario. Buenos Aires: Lajouane, 2007.

PRIEUR, M. Derecho del medio ambiente. 5. ed. Paris: Dalloz-Sirey, 2003.

SALINAS, J. Innovación docente y uso de las TIC en la enseñanza universitaria. Universidad y Sociedad del Conocimiento, Catalunya, v. 1, n. 1, 2004. Disponible en: < http://www.uoc.edu/rusc/dt/ esp/salinas1104.pdf>. Acesso em: 23 mar. 2017.

SANZ LARRUGA, F. Documentos y archivos electrónicos. España: 1Thonson Civitas, 2009.

SANTIAGO CAMPIÓN, R. Internet y enseñanza universitaria: de la gestión del conocimiento a la gestión del aprendizaje. 2001. Disponible en: <http://congresosdelalengua.es/valladolid/ponencias/ nuevas_fronteras_del_espanol/3_la_universidad_e_internet/campion_r.htm>. Acesso em: 23 mar. 2017. SAEZ VACAS, F. Cultura y tecnología en el nuevo entorno tecnosocial. Madrid: V.A, 2011.

SOLER, Yolanda; LEZCANO, M. Curso de Redacción Científica e Infotecnología. Revista Cognición. n. 26, 2011.

SOLER, Y. et al. La Web 2.0 en la producción de contenidos para la capacitación del profesional veterinario. RedVet, España, v. 16, n.11, p. 1-9, 2015.

STABLE-GUTIÉRREZ, C. Impacto de la infotecnología en el proceso investigativo. ICIDCA, Ciudad de La Habana, v. XL, n. 2, p.18-26, 2006. Disponível em: <http://www.redalyc.org/pdf/2231/223120664003. pdf> Acesso em: 23 mar. 2017.

UNESCO. Conferencia Mundial de Educación Superior 2009: Las nuevas dinámicas de la educación superior y de la investigación para el cambio social y el desarrollo. Paris, 2009.

TEARE, R.; Davies Sandelands, E. Organizaciones que aprenden formación virtual. Barcelona: Gedisa, 2002. 
TAELI, R. Los aportes de paradigmas complejos y constructivistas para la enseñanza de la ciencia jurídica. lus et Praxis, Talca, v. 20, n. 1, p. 199-220, 2014

TESORO, J. La Carta Iberoamericana de Gobierno Electrónico como hito hacia un nuevo paradigma de e-gobierno. España, 2008. Disponível em: <http://www.gobiernoelectronico.org/ node/5923>. Acesso em: 23 mar. 2017.

NACIONES UNIDAS. Boletin ONU no 13/108. Ciudad de México: Naciones Unidas, 2003. Disponível em: <http://www.cinu.org.mx/prensa/comunicados/2003/PR03108_Inf_e_gov.htm>. Acesso em: 23 mar. 2017.

INFANTE UGARTE, J. La empresa estatal socialista cubana y su transformación competitiva. Revista electrónica Cofín Habana, La Habana, n. 4, p. 84-94, 2014.

RAMOS, J.; RODRÍGUEZ, C. Cambio Tecnológico y Hegemonia Económica: el papel de las TICs en la Configuración del Nuevo Orden Económico Mundial. Revista de Estudios de Jubentud, Madrid, n. 108, p. 89-102, jun. 2015.

RICO CARRILLO, M. Derecho de las nuevas tecnologías. Buenos Aires: La Rocca, 2007.

RODRÍGUEZ, R. La auditoría ambiental en la gestión empresarial. Holguín: Colciencias, 2016.

REBOLLO PUIG, M. La actividad inspectora. España: lustel, 2011.

REVUELTA PÉREZ, I. Las nuevas tendencias en la inspección ambiental de las actividades industriales. Revista de Derecho Urbanístico y Medio Ambiente, Madrid, n. 185, p. 111-156, 2001.

SÁNCHEZ RODRÍGUEZ, F. Las nuevas tecnologías de la información y la comunicación en la Administración Pública como paradigmas de un servicio público eficaz y eficiente prestado a los ciudadanos. En: PUNZÓN MORALEDA, J. Administraciones públicas y nuevas tecnologías. Valladolid: Editorial Lex Nova, 2005.

JERÓNIMO MONTES, J.A. Hacia las comunidades virtuales de aprendizaje, aprender para apropiarse de los nuevos medios digitales. Revista Electrónica Teoría de la Educación. Educación y Cultura en la Sociedad de la Información, España, n. 2, p.246-262, 2009. Referência incompleta: cidade de publicação, volume

KUHN, T. Segundos pensamientos sobre paradigmas. Madrid: Fondo de Cultura Económica, 1975. KUHN, T. La estructura de las revoluciones científicas. México: Fondo de Cultura Económica, 1995. ZOROB AVILA, R. Estrategia curricular para la formación de la competencia de emprendimiento en negocio en redes universitarias. Revista Píxel-Bit de Medios de Comunicación, España, n. 41, p .149161, 2012.

VÁZQUEZ, B. El uso de aplicaciones para celulares como experiencia de Mobile learning en Ciencias Agrarias. IV Taller Internacional de Educación a Distancia y Pedagogía. Bayamo, 2015.

VALERO TORRIJOS, J. Régimen jurídico de la e-Administración: el uso de medios informáticos y telemáticos en el procedimiento administrativo. Granada: Comares, 2004.

VALVERDE BERROCOSO, I. El software libre y las "buenas prácticas" educativas con TIC, Comunicación y Pedagogía. Revista de Nuevas Tecnologías y Recursos Didácticos, España, n. 222, p. 48-55, 2007.

VERDES CABARCOS, Y. Tesis de Maestría: las etiquetas ambientales. Régimen jurídico del etiquetado ecológico. España: Universidad de la Coruña, 2014.

VIVES, A. La responsabilidad social de la empresa en América Latina. Washington: Fondo Multilateral de Inversiones, 2011.

Recebido em: 26/06/2017

Aprovado em: 20/09/2017 